\title{
ÉPOCAS DE CORTE E CULTIVARES NA COMPOSIÇÃO MINERAL DE FENO DE SOJA [Glycine max (L.) Merrill]
}

\author{
Cutting periods and cultivars in mineral composition of soybean hay [Glycine max (L.) Merril] \\ Cristiane Fortes Gris ${ }^{1}$, Pedro Milanez de Rezende ${ }^{2}$, Eudes de Arruda Carvalho ${ }^{3}$, \\ Élberis Pereira Botrel ${ }^{2}$, Antônio Ricardo Evangelista ${ }^{4}$, Messias José Bastos de Andrade ${ }^{2}$
}

\begin{abstract}
RESUMO
Com o objetivo de verificar a influência da época de corte sobre a composição mineral do feno de duas cultivares de soja foi conduzido ensaio em Lavras, MG, ano agrícola 2001/02. Utilizou-se o delineamento experimental de blocos casualizados, em esquema fatorial 2 x 5, com três repetições, compreendendo duas cultivares MG/BR46(Conquista) e M-SOY 8400 e cinco épocas de corte (estádios R2, R3, R4, R5 e R6). As épocas de corte influenciaram significativamente todos os nutrientes avaliados, tendo nos acúmulos de $\mathrm{P}$ e K sido detectada interação significativa de épocas de corte e cultivares. De maneira geral para todos os nutrientes à medida que se retardaram as épocas de corte observaram-se acréscimos nos acúmulos dos minerais. A cultivar M-SOY 8400 se destacou em relação a cultivar MG/BR46(Conquista) para acúmulo dos nutrientes $\mathrm{K}$ e Zn sendo a melhor opção para produção de feno. Em função dos resultados, pode-se inferir que o estádio de desenvolvimento R4 foi o que apresentou a melhor opção de corte para fenação, apresentando os maiores acúmulos de nutrientes, além da possibilidade de se liberar a área mais cedo.
\end{abstract}

Termos para indexação: Soja, minerais, feno.

\section{ABSTRACT}

The aim of the present investigation was to verify the influence of cutting periods on mineral composition of soybean hay. The experiment was conducted in Lavras, MG, Brazil, in the agricultural year 2001/02, on a typical Dystrophic Latosol. An experimental design in casualized blocks in factorial scheme $2 \times 5$ with three repetitions was employed, with two soybean cultivars MG/BR 46 (Conquista) and M-Soy 8400, and five cutting periods (stages R2, R3, R4, R5 and R6). Cutting periods have strongly influenced all the evaluated minerals, and significant interaction between cutting times and cultivars have been detected in $\mathrm{P}$ and $\mathrm{K}$ accumulation. On the whole, as cutting period was delayed, all minerals showed higher accumulation. Cultivar M-Soy 8400 showed higher $\mathrm{K}$ and $\mathrm{Zn}$ accumulation than MG/BR 46 (Conquista), standing out as the best option for hay production. According to the experimental results, development stage R4 presented the best cutting period for hay production, providing higher mineral accumulation and earlier clearance of harvest area.

Index terms: Soybean, minerals, hay.

(Recebido em 4 de abril de 2006 e aprovado em 9 de abril de 2007)

\section{INTRODUÇÃO}

Os minerais desempenham papel importante no organismo animal, sendo indispensáveis para sua manutenção e produção. Níveis deficientes de qualquer um dos 15 elementos considerados essenciais para o animal podem limitar o consumo e a utilização da forragem ingerida. As concentrações médias dos nutrientes encontrados nos tecidos vegetais são utilizadas como referência para mostrar o potencial de sua extração, translocação e obtenção de forrageiras com elevados níveis de produção e qualidade nutricional.
O conhecimento da variação da composição química, nas diversas fases do ciclo vegetativo, é um dos fatores a ser considerado para um adequado manejo. Diversos trabalhos demonstram que com o aumento da idade da planta os teores de alguns nutrientes decrescem em relação aos primeiros estádios (GIRON-CEDENO, 2001; PALHANO, 1990; SOEST et al., 1978; SOUZA FILHO, 1987).

Em virtude do estádio de desenvolvimento da planta apresentar ampla relação com sua composição química e qualidade forrageira, tornam-se imprescindíveis pesquisas no sentido de se identificar e quantificar as variações que cada espécie e/ou cultivar apresenta ao longo do ciclo,

\footnotetext{
'Engenheira Agrônoma, Doutoranda em Fitotecnia - Departamento de Agricultura/DAG - Universidade Federal de Lavras/UFLA - Cx. P. 3037 37200-000 - Lavras, MG - cristianegris@yahoo.com.br

${ }^{2}$ Engenheiros Agrônomos, Doutores, Professores - Departamento de Agricultura/DAG - Universidade Federal de Lavras/UFLA - Cx. P. 3037 - 37200 000 - Lavras, MG - pmrezend@ufla.br; elberis@ufla.br; mandrade@ufla.br

${ }^{3}$ Engenheiro Agrônomo, Mestrando em Fitotecnia - Departamento de Fitopatologia/DFP - Universidade Federal de Lavras/UFLA - Cx. P. 3037 37200-000 - Lavras, MG - eudes@agronomia.ufla.br

${ }^{4}$ Engenheiro Agrônomo, Doutor, Professor Departamento de Zootecnia/DZO - Universidade Federal de Lavras/UFLA - Cx. P. 3037 - $37200-000$ -

Lavras, MG - aricardo@ufla.br
} 
determinando-se assim a fase de desenvolvimento em que a planta apresenta o máximo valor nutricional (GOMES, 2003).

No caso específico das leguminosas, em trabalhos com a utilização de diferentes cultivares de soja para produção de feno, observou-se variações dentro da espécie quanto à composição química da forragem (BOTREL et al., 2003; REZENDE \& CARVALHO, 1992; REZENDE et al., 1997a,b, 2004; SILVA et al., 2003), embora não exista na mesma proporção estudos que quantifiquem tais diferenças à medida que o ciclo da cultura avança (OLIVEIRA \& REZENDE, 1987; REZENDE \& CARVALHO, 1992; REZENDE \& TAKAHASHI, 1990; REZENDE et al., 2001).

Segundo compilação feita por Silva (1998), observase que forragem de boa qualidade pode ser obtida a partir do início da formação das vagens até o amarelecimento inicial das folhas em plantas de soja. Segundo Santos \& Vieira (1982) a parte aérea da planta de soja, quando cortada até o estádio V13 e fenada, tem aproximadamente o mesmo valor nutritivo de outras leguminosas forrageiras como alfafa e soja perene.

Oliveira \& Rezende (1987), também evidenciaram com seus ensaios que cortes mais tardios para produção de feno de soja, por volta dos 75 e 90 dias, proporcionaram redução na qualidade da forragem, e que o corte efetuado aos 60 dias proporcionou maiores acúmulos dos minerais potássio, cálcio e magnésio. Munhoz et al. (1983) relata que para se manter um ótimo balanço entre a qualidade do feno produzido e seu rendimento, o ideal é que o corte seja realizado nas fases de formação e enchimento de vagens, compreendidas durantes os estádios reprodutivos R2 e R6.

Oliveira (1987) em estudo sobre o efeito da época de corte sobre o rendimento de feno e grãos oriundos da rebrota para a cultivar Cristalina, observou queda nos teores de $\mathrm{P}, \mathrm{K}$ e Ca para a idade de corte de 90 dias em relação as idades de 60 e 75 dias, sendo o $\mathrm{Mg}$ aos 75 e 90 dias iguais entre si e superiores àqueles obtidos aos 60 dias. Blank \& Rezende (1994) utilizando as cultivares de soja IAC 8, Doko e Cristalina, cortadas ao 60 dias para produção de feno e grãos de rebrota, verificou-se semelhanças entre o feno de soja e alfafa quando comparados os valores médios de cálcio e fósforo.

A necessidade de maiores informações sobre a utilização da soja para fenação, sobretudo no que diz respeito às recomendações de cultivares, bem como as melhores épocas de corte, representam ainda uma grande demanda do setor pecuário. Dentro deste contexto, objetivou-se com este trabalho avaliar a composição mineral de cultivares e épocas de corte para produção de feno de soja [Glycine max (L.) Merrill].

\section{MATERIAL E MÉTODOS}

O ensaio foi conduzido no Campus Experimental da Universidade Federal de Lavras - UFLA, município de
Lavras (MG), situado a uma altitude de $900 \mathrm{~m}$ e instalado em 02/12/01 em solo classificado como Latossolo Roxo Distrófico de textura argilosa, fase cerrado, cujas características foram as seguintes: $\mathrm{pH}$ em água 5,8 ACM ; $\mathrm{Al}^{+++}$trocável $\left(\mathrm{cmoldm}^{-3}\right)=0,3 \mathrm{~B} ; \mathrm{Ca}^{++}\left(\mathrm{cmoldm}^{-3}\right)=2,2 \mathrm{~B}$; $\mathrm{Mg}^{++}\left(\mathrm{cmoldm}^{-3}\right)=0,5 \mathrm{M} ; \mathrm{K}^{+}\left(\mathrm{mgdm}^{-3}\right)=27 \mathrm{~B} ; \mathrm{P}\left(\mathrm{mgdm}^{-3}\right)=12$ Bom (sendo $\mathrm{ACM}=$ acidez média, $\mathrm{B}=$ baixo), seguindo interpretações de Ribeiro et al. (1999).

A adubação de semeadura foi feita de acordo com a análise de solo e as interpretações de acordo com Ribeiro et al. (1999), utilizando-se $400 \mathrm{~kg} \cdot \mathrm{ha}^{-1}$ do formulado 04-30-10. As sementes de soja foram inoculadas antes da semeadura com Bradyrhizobium japonicum, utilizando o inoculante turfoso Nitral na proporção de $200 \mathrm{~g}$ para $50 \mathrm{~kg}$ de sementes. Todos os tratamentos receberam sempre que necessário os tratos culturais indispensáveis à cultura.

O delineamento experimental foi o de blocos casualizados com três repetições em esquema fatorial $2 \times 5$, compreendendo duas cultivares de soja MG/BR 46 (Conquista) e M-SOY 8400 e cinco épocas de corte, realizadas nos estádios fenológicos R2, R3, R4 ,R5, e R6, de acordo com Fehr \& Caviness (1977).

As parcelas foram constituídas de quatro fileiras de 5,0m, espaçadas de $0,5 \mathrm{~m}$, mantendo densidade de 25 pl. $\mathrm{m}^{-1}$, sendo avaliadas como parcelas úteis as duas fileiras internas. Os cortes foram realizados rente ao solo, com o auxílio de uma roçadeira costal motorizada.

A composição mineral do feno de soja foi determinada a partir de uma amostra de $300 \mathrm{~g}$ da matéria seca total, obtida por meio do corte das parcelas uteis e enviada ao Laboratório de Análise Foliar da UFLA, quantificando-se os teores dos nutrientes $\mathrm{P}, \mathrm{K}, \mathrm{Ca}, \mathrm{Mg}, \mathrm{S}$, $\mathrm{Cu}, \mathrm{Mn}$ e $\mathrm{Zn}$. A partir dos resultados obtidos, esses foram calculados em função do feno e expressos em $\mathrm{kg} \mathrm{ha}^{-1}$ para macrominerais e $\mathrm{g} \mathrm{ha}^{-1}$ para os microminerais.

As análises de variância para todas as características avaliadas foram realizadas por meio do programa estatístico SISVAR ${ }^{\circledR}$, segundo Ferreira (2000) e as médias comparadas pelo teste de Scott-Knott ao nível de $5 \%$ de probabilidade.

\section{RESULTADOS E DISCUSSÃO}

Detectou-se através das análises de variância que o fator de variação épocas de corte alterou significativamente todas as características avaliadas, tendo a interação cultivar e época de corte apresentado significância somente para os acúmulos de $\mathrm{P}$ e K (Tabelas 1 e 2). Para todos os minerais avaliados o corte realizado em R2 apresentou os menores acúmulos, diferindo-se de todas as demais épocas de corte. 
Tabela 1 - Resumo da análise de variância para os acúmulos de P, K, Ca, Mg e S no feno, obtidos no ensaio cultivares e épocas de corte para produção de feno de soja, Lavras, MG, 2002.

\begin{tabular}{lcccccc}
\hline \multirow{2}{*}{ Causa de variação } & \multirow{2}{*}{ G.L } & \multicolumn{5}{c}{ Quadrados Médios } \\
\cline { 4 - 7 } & & $\mathrm{P}$ & $\mathrm{K}$ & $\mathrm{Ca}$ & $\mathrm{Mg}$ & $\mathrm{S}$ \\
\hline Blocos & 2 & 4,78 & 619,70 & 486,66 & 26,07 & 34,57 \\
Cultivares (C) & 1 & $10,53^{\mathrm{NS}}$ & $3410,56^{* *}$ & $8,65^{\mathrm{NS}}$ & $2,40^{\mathrm{NS}}$ & $0,63^{\mathrm{NS}}$ \\
Épocas (E) & 4 & $143,91^{* *}$ & $6264,78^{* *}$ & $9659,12^{* *}$ & $82,28^{* *}$ & $128,23^{* *}$ \\
C x E & 4 & $19,83^{* *}$ & $802,92^{*}$ & $589,40^{\mathrm{NS}}$ & $3,952^{\mathrm{NS}}$ & $3,60^{\mathrm{NS}}$ \\
Resíduo & 18 & 3,09 & 215,68 & 375,67 & 4,73 & 4,25 \\
\hline CV(\%) & 12,14 & 11,48 & 14,86 & 13,44 & 11,46 \\
\hline
\end{tabular}

* Significativo pelo teste "F" a $5 \%$ de probabilidade; ** significativo pelo teste "F" a $1 \%$ de probabilidade; ${ }^{N S}$ não significativo pelo teste " $F$ ".

Tabela 2 - Resumo da análise de variância para os acúmulos de $\mathrm{Cu}$, Mn e Zn no feno, obtidos no ensaio cultivares e épocas de corte para produção de feno de soja, Lavras, MG, 2002.

\begin{tabular}{lcccc}
\hline \multirow{2}{*}{ Causa de variação } & \multirow{2}{*}{ G.L. } & \multicolumn{3}{c}{ Quadrados Médios } \\
\cline { 3 - 5 } Blocos & 2 & $\mathrm{Cu}$ & $\mathrm{Mn}$ & 3469,92 \\
Cultivares (C) & 1 & $0,14^{\mathrm{NS}}$ & 7455,65 & $14597,98^{* *}$ \\
Épocas (E) & 4 & $1502,37^{* *}$ & $300,77^{\mathrm{NS}}$ & $52534,01^{* *}$ \\
C x E & 4 & $176,61^{\mathrm{NS}}$ & $127612,21^{* *}$ & $3558,63^{\mathrm{NS}}$ \\
Resíduo & 18 & $76,41^{\mathrm{NS}}$ & $1995,63^{\mathrm{NS}}$ & 500,68 \\
\hline CV(\%) & & 16,08 & 2880,91 & 10,96 \\
\hline
\end{tabular}

** Significativo pelo teste "F" a 1\% de probabilidade; Ns não significativo pelo teste "F".

De maneira geral pode-se observar que para todos os minerais avaliados, à medida que os cortes foram realizados em estádios mais avançados observou-se acréscimos significativos nos acúmulos desses. Tais resultados vêm de encontro aos obtidos por Rezende et al. (2001) que observaram acréscimos significativos nos acúmulos de $\mathrm{P}, \mathrm{K}, \mathrm{Ca}$ e $\mathrm{Mg}$ com o atraso dos cortes.

\section{Fósforo e Potássio}

Observou-se interação significativa entre cultivares e épocas de corte somente para os minerais fósforo e potássio (Tabela 1). Quando analisados os resultados obtidos para a cultivar MG/BR 46 (Conquista) em relação ao acúmulo de fósforo, verificou-se que os maiores valores foram obtidos quando os cortes foram realizados nos estádios R4 e R6, o que difere um pouco do comportamento da cultivar M-SOY 8400 que apresentou seus melhores acúmulos nos últimos dois estádios R5 e R6 (Tabela 3). Resultados de Cardoso (1985) se assemelham aos obtidos neste trabalho quanto aos acúmulos de fósforo.

Quanto aos acúmulos de potássio em função dos cortes, verificou-se que para a cultivar MG/BR 46
(Conquista) os maiores valores ocorreram a partir do estádio R3, não tendo se observado a partir desta época diferenças significativas entre as épocas, o que difere do comportamento da cultivar M-SOY 8400 que apresentou maiores acúmulos desses mineral no corte R6. Pode-se inferir ainda que a absorção de potássio pela cultivar MG/BR 46 (Conquista) ocorre em menor proporção quando comparada com os valores absolutos acumulados para a cultivar M-SOY 8400. O acúmulo máximo para a primeira cultivar ocorre somente em R6 enquanto que para a cultivar M-SOY 8400 isto se dá já em R3, mostrando-se esta última mais eficiente na absorção deste mineral. Comparando-se as cultivares quanto aos respectivos acúmulos de potássio, a cultivar M-SOY 8400 supera a cultivar MG/BR 46 (Conquista) em $18,17 \%\left(21,32 \mathrm{~kg} \mathrm{ha}^{-1}\right)$.

Estes resultados comprovam a grande variabilidade existente entre materiais genéticos, implicando respostas diferenciais não só em sua composição química como proteína, mas também para os diversos minerais acumulados durante o ciclo da cultura. Resultados observados em diversos outros trabalhos também evidenciam o efeito diferencial de cultivares, quando 
avaliada a composição mineral do feno (REZENDE \& TAKAHASHI, 1990; REZENDE et al., 1997a,b). Segundo Amaral (1981) e Oliveira (1981), a soja é uma importante fonte de minerais como potássio e cálcio, com destaque para o elevado teor de potássio, cerca de $2 \%$.

\section{Cálcio, Magnésio e Enxofre}

Da mesma maneira que ocorreu para fósforo e potássio, também observou-se para cálcio, magnésio e enxofre acúmulos a medida que os cortes foram realizados em estádios mais avançados, atingindo os maiores valores à partir de R5 para o cálcio e R4 para magnésio e enxofre (Tabela 4).

Rezende et al. (2001) em estudo realizado nesta mesma região, também observaram acréscimos significativos nos acúmulos de cálcio e magnésio quando os cortes foram realizados em estágios mais avançados. Resultados de Oliveira (1987) e Rezende (1984) se assemelham aos obtidos neste presente trabalho para os acúmulos de cálcio.

Os resultados obtidos neste trabalho eram de certa forma esperados, pois com o avançar do ciclo, as plantas já se encontravam no estádio final de desenvolvimento e, consequentemente, já haviam acumulado a maior parte desses minerais que, de acordo com Cordeiro et al. (1979) ocorrem aos 59 e 82 dias após emergência para Ca e P, respectivamente.

Todos os minerais avaliados apresentaram aumentos em seus acúmulos no feno à medida que os cortes foram realizados em estádios mais avançados. Segundo Norton (1982) o caráter imóvel do enxofre na planta faz com que sua concentração varie muito. Esse elemento é requerido pelas plantas em quantidades semelhantes ao fósforo, uma vez que a tendência do enxofre é reagir com outros componentes, fato que faz com que o mesmo seja muito mais disponível que o fósforo às plantas, conforme relata Vale et al. (1997).

\section{Cobre, Manganês e Zinco}

A análise de variância detectou efeito significativo das épocas de corte sobre os acúmulos dos microminerais cobre, manganês e zinco, sendo que somente para o zinco ocorreu efeito significativo de cultivares (Tabela 2).

Os resultados médios obtidos para cobre, manganês e zinco podem ser visualizados na Tabela 4. Em relação aos microminerais, as épocas de corte R4, R5 e R6 apresentaram os maiores acúmulos para cobre, e as épocas R5 e R6 para manganês e enxofre.

Em relação às cultivares testadas verifica-se que no caso do $\mathrm{Zn}$ ocorreu uma resposta diferencial. A cultivar M-SOY 8400 superou a cultivar MG/BR 46 (Conquista) proporcionando um aumento de $44,11 \mathrm{~g} \mathrm{ha}^{-1}$ $(24,21 \%)$. Segundo Costa et al. (1974) e Smith \& Circle (1972), a variação nos teores de minerais pode estar relacionada às condições de cultivo, clima, solo e adubação, o que faz com que algumas diferenças sejam encontradas em avaliações de minerais em ensaios experimentais.

Tabela 3 - Resultados médios para os acúmulos de $\mathrm{P}$ e $\mathrm{K}$ no feno, obtidos no ensaio cultivares e épocas de corte para produção de feno de soja, Lavras, MG, 2002.

\begin{tabular}{|c|c|c|c|c|c|c|}
\hline \multirow{3}{*}{ Época de corte } & \multicolumn{6}{|c|}{ Acúmulo $\left(\mathrm{kg} \cdot \mathrm{ha}^{-1}\right)$} \\
\hline & \multicolumn{3}{|c|}{$\mathrm{P}$} & \multicolumn{3}{|c|}{$\mathrm{K}$} \\
\hline & $\begin{array}{l}\text { MG/BR46 } \\
\text { Conquista }\end{array}$ & $\begin{array}{c}\text { M-SOY } \\
8400\end{array}$ & Média & $\begin{array}{l}\text { MG/BR46 } \\
\text { Conquista }\end{array}$ & $\begin{array}{c}\text { M-SOY } \\
8400\end{array}$ & Média \\
\hline $\mathrm{R} 2$ & $7,40 \mathrm{c}$ & $6,65 \mathrm{c}$ & $7,02 \mathrm{~d}$ & $63,03 \mathrm{~b}$ & $77,71 \mathrm{c}$ & $70,37 \mathrm{~d}$ \\
\hline R3 & $9,39 \mathrm{c}$ & $9,14 \mathrm{c}$ & $9,26 \mathrm{c}$ & $96,07 \mathrm{a}$ & $88,62 \mathrm{c}$ & $92,35 \mathrm{c}$ \\
\hline R4 & 14,36 a & $12,61 \mathrm{~b}$ & $13,49 \mathrm{~b}$ & $107,67 \mathrm{a}$ & $123,13 \mathrm{~b}$ & $115,40 \mathrm{~b}$ \\
\hline R5 & $11,39 \mathrm{~b}$ & $17,43 \mathrm{a}$ & $14,41 \mathrm{~b}$ & 116,89 a & $137,24 \mathrm{~b}$ & $127,06 \mathrm{a}$ \\
\hline R6 & $16,56 \mathrm{a}$ & $18,31 \mathrm{a}$ & $17,44 \mathrm{a}$ & $114,88 \mathrm{a}$ & 162,48 a & $138,68 \mathrm{a}$ \\
\hline Média & $11,82 \mathrm{~A}$ & $12,82 \mathrm{~A}$ & 12,32 & 99,71 B & $117,83 \mathrm{~A}$ & 108,77 \\
\hline
\end{tabular}

Médias seguidas pela mesma letra minúscula na coluna e maiúscula na linha não diferem entre si, pelo teste "Scott-Knott" a $5 \%$ de probabilidade. 
Tabela 4 - Resultados médios para o acúmulo dos minerais $\mathrm{Ca}, \mathrm{Mg}, \mathrm{S}\left(\mathrm{kg} \mathrm{ha}^{-1}\right) \mathrm{Cu}, \mathrm{Mn}$ e $\mathrm{Zn}\left(\mathrm{g} \mathrm{ha}^{-1}\right)$ no feno, obtidos no ensaio de cultivares e épocas de corte para produção de feno de soja, Lavras, MG, 2002.

\begin{tabular}{crrrrrr}
\hline Época de corte & \multicolumn{5}{c}{ Acúmulo } \\
\cline { 2 - 7 } & $\mathrm{Ca}$ & $\mathrm{Mg}$ & $\mathrm{S}$ & $\mathrm{Cu}$ & $\mathrm{Mn}$ & $\mathrm{Zn}$ \\
\hline R2 & $66,72 \mathrm{c}$ & $9,25 \mathrm{c}$ & $8,92 \mathrm{c}$ & $27,07 \mathrm{c}$ & $186,77 \mathrm{~d}$ & $88,63 \mathrm{c}$ \\
R3 & $85,83 \mathrm{c}$ & $12,01 \mathrm{~b}$ & $14,16 \mathrm{~b}$ & $38,02 \mathrm{~b}$ & $248,31 \mathrm{c}$ & $101,81 \mathrm{c}$ \\
R4 & $116,30 \mathrm{~b}$ & $14,65 \mathrm{a}$ & $17,17 \mathrm{a}$ & $50,59 \mathrm{a}$ & $373,88 \mathrm{~b}$ & $170,30 \mathrm{~b}$ \\
R5 & $141,12 \mathrm{a}$ & $15,69 \mathrm{a}$ & $18,38 \mathrm{a}$ & $55,60 \mathrm{a}$ & $441,26 \mathrm{a}$ & $246,21 \mathrm{a}$ \\
R6 & $144,52 \mathrm{a}$ & $17,18 \mathrm{a}$ & $17,90 \mathrm{a}$ & $59,71 \mathrm{a}$ & $475,30 \mathrm{a}$ & $261,04 \mathrm{a}$ \\
\hline MG/BR 46 & $110,44 \mathrm{a}$ & $13,51 \mathrm{a}$ & $15,18 \mathrm{a}$ & $46,14 \mathrm{a}$ & $342,41 \mathrm{a}$ & $154,85 \mathrm{~b}$ \\
(Conquista) & $111,36 \mathrm{a}$ & $14,00 \mathrm{a}$ & $15,43 \mathrm{a}$ & $46,26 \mathrm{a}$ & $347,79 \mathrm{a}$ & $192,34 \mathrm{a}$ \\
M-SOY 8400 & 110,90 & 13,75 & 15,31 & 47,05 & 345,10 & 173,60 \\
\hline Médias & &
\end{tabular}

Médias seguidas pela mesma letra na coluna não diferem, pelo teste "Scott-Knott" a $5 \%$ de probabilidade.

\section{CONCLUSÕES}

A cultivar de soja M-SOY 8400 destacou-se em relação a cultivar MG/BR 46 (Conquista) em virtude de maiores acúmulos de $\mathrm{K}$ e $\mathrm{Zn}$.

O estádio de desenvolvimento R4 apresentou a melhor opção de corte para fenação, independente da cultivar.

Para todos os minerais avaliados, em ambas cultivares, cortes em estádios mais avançados proporcionaram acréscimos em seus acúmulos.

\section{REFERÊNCIA BIBLIOGRÁFICA}

AMARAL, A. Alimentação racional. In: MIYASAKA, S.; MEDINA, J. C. (Eds.). A soja no Brasil. Campinas: ITAL, 1981. p. 832-839.

BLANK, A. F.; REZENDE, P. M. de. Efeito da adubação nitrogenada no plantio sobre produção e características químicas do feno de soja [Glycine Max (L.) Merrill]. Ciência e Prática, Lavras, v. 18, n. 3, p. 258-263, jul./set. 1994.

BOTREL, E. P.; REZENDE, P. M. de; EVANGELISTA, A. R.; MORAES, A. R. Avaliação do rendimento forrageiro da soja em quatro sistemas de corte, sucedida por milheto ou milho. Ciência e Agrotecnologia, Lavras, v. 27, n. 5, p. 1122-1129, set./out. 2003.

CARDOSO, D. A. del B. Maximização da exploração da soja (Glycine max (L.) MERRILL): efeito do espaçamento, densidade e altura de corte na produção de feno e grãos da rebrota, cv. Cristalina. 1985. 83 f. Dissertação (Mestrado) - Universidade Federal de Lavras, Lavras, 1985.
CORDEIRO, D. S.; SFREDO, D. J.; BORKERT, L. M.; SARRUGE, J. R.; PALHANO, J. B.; CAMPO, R. J. Calagem, adubação e nutrição mineral. In: EMPRESA BRASILEIRA DE PESQUISA AGROPECUÁRIA. Centro Nacional de Pesquisa de Soja. Ecologia, manejo e adubação de soja. Londrina: UFPR, 1979. cap. 2, p. 19-62. (Circular técnica, 2).

COSTA, S. I.; MIYA, E. E.; FUJITA, J. T. Composição química e qualidades organolépticas e nutricionais das principais variedades de soja cultivadas no Estado de São Paulo. Coletânea do Instituto de Tecnologia de Alimentos, Campinas, v. 5, p. 305-319, 1974.

FEHR, W. R.; CAVINESS, C. E. Stage of soybean development. Iowa: Ames, 1977. 11 p.

FERREIRA, D. F. Análises estatísticas por meio do Sisvar para Windows versão 4.0. In: REUNIÃO ANUAL DA REGIÃO BRASILEIRA DA SOCIEDADE INTERNACIONAL DE BIOMETRIA, 2000, São Carlos. Resumos... São Carlos: RBRAS/UFSCar, 2000. p. 255258.

GIRON CEDENO, J. A. Estudo de gramíneas tropicais em diferentes idades. 2001. 66 p. Dissertação (Mestrado) Universidade Federal de Lavras, Lavras, 2001.

GOMES, V. M. Disponibilidade e valor nutritivo de brachiaria vedada para uso na região semi-árida de Minas Gerais. 2003. 99 p. Dissertação (Mestrado) - Universidade Federal de Lavras, Lavras, 2003. 
MUNOZ, A. E.; HOLT, E. C.; WEAVER, R. W. Yield and quality of soybean hay as influenced by stage of growth and plant density. Agronomy Journal, Madison, v. 75, n. 1, p. 147-149, 1983.

NORTON, B. W. Differences in plant species in forage quality. In: INTERNATIONAL SYMPOSIUM ON NUTRITIONAL LIMITRS TO ANIMAL PRODUCTION FROM PASTURES, 1981, Saint Lucia. Proceedings... Farnham Royal: Commonwealth Agricultural Bureaux, 1982. p. $89-110$.

OLIVEIRA, J. E. D. de. Valor da soja como alimento. In: MIYASAKA, S.; MEDINA, J. C. (Eds.). A soja no Brasil. Campinas: ITAL, 1981. p. 820-823.

OLIVEIRA, J. N. S.; REZENDE, P. M. de. Maximização da exploração da soja [Glycine max (L.) Merrill]: efeito da época de corte e adubação nitrogenada na produção de feno e grãos oriundos da rebrota, cv. Cristalina. Ciência e Prática, Lavras, v. 11, n. 1, p. 65-74, 1987.

OLIVEIRA, J. R. da C. Produção, composição química e digestibilidade da gramínea Hemarthriz altissima (POIR) Stapf e Hubbard cv. Bigalta em diferentes idades de corte. 1987. 70 f. Dissertação (Mestrado) - Universidade Federal de Lavras, Lavras, 1987.

PALHANO, A. L. Recrutamento de nutrientes e valor nutritivo de Cynodon dactylon (L.) Pers. Cv. Coastcross no 1. 1990. 122 f. Dissertação (Mestrado) - Escola Superior de Agricultura de Luiz de Queiroz, Piracicaba, 1990.

REZENDE, P. M. de. Maximização da exploração da soja: I. efeito do corte aos 60 dias na produção de feno e grãos da rebrota. Pesquisa Agropecuária Brasileira, Brasília, v. 19, n. 3, p. 329-336, 1984.

REZENDE, P. M. de; ANDRADE, M. J. B. de; RESENDE, G. M.; BOTREL, E. P. Maximização da exploração da soja [Glycine $\max ($ L.) Merrill]: efeito da época de corte e da adubação fosfatada na produção de feno e grãos da rebrota. Ciência e Agrotecnologia, Lavras, v. 25, n. 2, p. 299-310, mar./abr. 2001.

REZENDE, P. M. de; BLANK, A. F.; REZENDE, G. M. de. Maximização da exploração da soja [Glycine max (L.) Merrill]: efeito de sistemas de corte e cultivares na produção de feno. Ensaios e Ciência, Campo Grande, v. 1, n. 1, p. 131-141, dez. 1997a.
REZENDE, P. M. de; CARVALHO, E. R. de. Maximização da exploração da soja [Glycine max (L.) Merrill]: efeito de sistemas de corte, adubação nitrogenada no plantio e cultivares na produção de feno. Ciência e Prática, Lavras, v. 16, p. 260-269, abr./jun. 1992.

REZENDE, P. M. de; CARVALHO, E. R. de; REZENDE, G. M. de. Maximização da exploração da soja [Glycine max (L.) Merrill]: efeito de sistemas de corte e da adubação nitrogenada em cobertura na seleção de cultivares para produção de feno. Ciência e Agrotecnologia, Lavras, v. 21, n. 4, p. 457-464, out./dez. 1997b.

REZENDE, P. M.; SILVA, A. G.; CORTE, E.; BOTREL, E. P.; GOMES, L. L.; GRIS, C. F. Consórcio sorgo-soja: sistemas de corte na produção de forragens das culturas consorciados na entrelinha. Revista Brasileira de Agrociência, [S.1.], v. 10, n. 4, p. 475-481, 2004.

REZENDE, P. M. de; TAKAHASHI, S. Maximização da exploração da soja [Glycine max (L.) Merrill]: efeito do sistema de cortes na seleção de cultivares para produção de feno. Ciência e Prática, Lavras, v. 14, p. 44-55, jan./ abr. 1990 .

RIBEIRO, A. C.; GUIMARÃES, P. T. G.; VICENTE, V. H. A. Recomendações para o uso de corretivos e fertilizantes em Minas Gerais: $5^{\text {a }}$ aproximação. Lavras: UFLA, 1999. 359 p.

SANTOS, O. S. dos; VIEIRA, C. Crescimento e qualidade nutritiva da planta de soja [Glycine $\max (\mathrm{L}$.) Merrill]. Revista Ceres, Viçosa, v. 29, n. 161, p. 107-115, jan./mar. 1982.

SILVA, A. G. da. Produção de forragem de cultivares de sorgo e soja, consorciadas na linha, em dois sistemas de corte. Lavras: UFLA, 1998. 80 p.

SILVA, A. G.; REZENDE, P. M.; GRIS, C. F.; GOMES, L. L.; BOTREL, E. P. Consórcio sorgo-soja: influência de sistemas de cortes, na produção de forragens de sorgo e soja consorciados na linha e do sorgo em monocultivo. Ciência e Agrotecnologia, Lavras, v. 27, n. 2, p. 451-461, 2003.

SMITH, A. K.; CIRCLE, S. J. Chemical composition of the seed. In: Soybeans: chemistry and technology. Westport: The AVI, 1972. v. 1, chap. 3, p. 61-92. 
SOEST, P. J. van; MERTENS, D. R.; DEINUM, B. Preharvest factors influencing quality of conserved forage. Journal of Animal Science, Champaign, v. 47, n. 3, p. 712-720, 1978.

SOUZA FILHO, A. P. da S. Rendimento forrageiro, composição química e digestibilidade das frações folha $\mathrm{e}$ colmo do capim elefante (Pennisetum purpureum Scum. “Dwarf”') em diferentes idades. Lavras: ESAL, 1987. 104 p.

VALE, F. R. do; GUILHERMNE, L. R. G.; AQUINO-GUEDES, G. A. de; FURTINI NETO, A. E. Fertilidade do solo: dinâmica e disponibilidade de nutrientes. Lavras: UFLA/FAEPE, 1997. $171 \mathrm{p}$. 